Article

\title{
A $\tau$-Symmetry Algebra of the Generalized Derivative Nonlinear Schrödinger Soliton Hierarchy with an Arbitrary Parameter
}

\author{
Jian-bing Zhang ${ }^{1, * \mathbb{C}}$, Yingyin Gongye ${ }^{1}$ and Wen-Xiu Ma ${ }^{2,3,4}$ \\ 1 School of Mathematics and Statistics, Jiangsu Normal University, Xuzhou 221116, Jiangsu, China; \\ gongyeyingyin@163.com \\ 2 Department of Mathematics and Statistics, University of South Florida, Tampa, FL 33620, USA; \\ wma3@usf.edu \\ 3 College of Mathematics and Systems Science, Shandong University of Science and Technology, \\ Qingdao 266590, Shandong, China \\ 4 Department of Mathematical Sciences, North-West University, Mafikeng Campus, \\ Mmabatho 2735, South Africa \\ * Correspondence: jbzmath@jsnu.edu.cn; Tel.: +86-150-5082-5896
}

Received: 29 August 2018; Accepted: 22 October 2018; Published: 23 October 2018

\begin{abstract}
A matrix spectral problem is researched with an arbitrary parameter. Through zero curvature equations, two hierarchies are constructed of isospectral and nonisospectral generalized derivative nonlinear schrödinger equations. The resulting hierarchies include the Kaup-Newell equation, the Chen-Lee-Liu equation, the Gerdjikov-Ivanov equation, the modified Korteweg-de Vries equation, the Sharma-Tasso-Olever equation and a new equation as special reductions. The integro-differential operator related to the isospectral and nonisospectral hierarchies is shown to be not only a hereditary but also a strong symmetry of the whole isospectral hierarchy. For the isospectral hierarchy, the corresponding $\tau$-symmetries are generated from the nonisospectral hierarchy and form an infinite-dimensional symmetry algebra with the $K$-symmetries.
\end{abstract}

Keywords: generalized derivative nonlinear shcrödinger equation; master symmetry; infinitedimensional Lie algebra

\section{Introduction}

As we all know, from a matrix spectral problem, we can obtain isospectral and nonisospectral soliton hierarchies [1,2]. The inverse scattering transformation is one of most powerful tools for solving these equations [1,2]. Furthermore, these equations are integrable and they have a lot of integrable characteristics, including the existence of conservation laws (CLs) and infinitely many symmetries [3].

There are many approaches to seek the CLs for $(1+1)$-dimensional integrable systems, such as the method based on the non-semisimple Lie algebras to formulate generating functions for conserved densities by the variational identities [4,5], utilizing adjoint symmetries [6,7] and expanding the ratios of eigenfunctions of matrix spectral problems [8,9]. Lax pairs have been used in generating CLs for various evolution equations [8-12]. How to obtain a conservation density is very important from the spectral problem of Lax pairs. Actually, CLs can be derived by taking advantage of the generating conservation density and evolution equation of time [13].

It is another important integrable property that an evolution equation owns $K$-symmetries. We remark that these symmetries do not depend explicitly on space and time variables. Li and Zhu found the $\tau$-symmetries which depend explicitly on space variable by a general method in $1987[14,15]$. They further discovered that these symmetries form a infinite Lie algebra along with $K$-symmetries. 
It is interesting that, regarding $\tau$-symmetries as vector fields, we can also generate new symmetries for the evolution equations [16,17]. In fact, all these $\tau$-symmetries are produced from the first degree generators [18]. Later, based on the previous work, a more generalized framework was established on $K$-symmetries and $\tau$-symmetries of the evolution equations $[19,20]$ by one of the authors (Ma). At the same time, the infinite-dimensional symmetry Lie algebra was also discussed. In last few years, symmetries of discrete soliton hierarchies were also discussed [21-24].

As we know, there are three famous derivative nonlinear schrödinger (DNLS) equations including the Kaup-Newell (KN) equation, the Chen-Lee-Liu (CLL) equation and the Gerdjikov-Ivanov (GI) equation [25-29]. Starting from a matrix spectral problem with an arbitrary parameter, we generate two hierarchies of generalized derivative nonlinear schrödinger (gDNLS) equations including isospectral and nonisospectral hierarchies. The famous three isospectral and nonisospectral DNLS equations mentioned above are deduced by selecting special values of the arbitrary parameter. We show that the nonisospectral gDNLS hierarchy with an arbitrary parameter is the $\tau$-symmetries for the isospectral gDNLS hierarchy with the same parameter. Finally, we prove that the resulting $\tau$-symmetries constitute an infininte-dimensional Lie algebra with $K$-symmetries.

The paper is organized as follows. In Section 2, the basic notions and notations are discussed. In Section 3, two hierarchies of the isospectral and nonisospectral gDNLS hierarchies with an arbitrary parameter are presented. In Section 4 , the $K$-symmetries and $\tau$-symmetries are deduced, and the two kinds of symmetries form an infinite-dimensional Lie algebra. We conclude the paper in Section 5.

\section{Basic Notions}

First, we give some basic notions and notations used in this paper [19,24]. Let $\mathscr{L}$ be the set of differential vector functions which map $\mathbb{R}^{N} \times \mathbb{R} \times \mathcal{L}$ into $\mathcal{L}$, where $\mathbb{R}$ and $\mathbb{C}$ are the real and complex fields, respectively, and $\mathcal{L}$ is one linear topological space over $\mathbb{C}$.

Definition 1. Let $K=K(u)=K(x, t, u), S=S(u)=S(x, t, u) \in \mathscr{L}$. We define

$$
K^{\prime}[S]=K^{\prime}(u)[S(u)]=\left.\frac{\partial}{\partial \varepsilon} K(u+\varepsilon S(u))\right|_{\varepsilon=0}
$$

is the Gateaux derivative of $K(u)$ in the direction $S(u)$ with respect to $u$, where $K(u)=K(x, t, u)$ means $u$ is a function of variables $x, t$ and $K(x, t, u)$ is a certain function of $x, t, u$ and its $x$ derivatives, but we abbreviate $K(x, t, u)$ as $K(u)$ to emphasize $u$ and its $x$ derivatives.

As we know, with respect to the following product:

$$
\llbracket K, S \rrbracket=\llbracket K(u), S(u) \rrbracket=K^{\prime}(u)[S(u)]-S^{\prime}(u)[K(u)] \quad K, S \in \mathscr{L},
$$

$\mathscr{L}$ forms a Lie algebra. Suppose that the differentiable function or a differential vector function $u=u(x, t)$ map $\mathbb{R}^{N} \times \mathbb{R}$ into $\mathcal{L}$. We take into account the following evolution equation

$$
u_{t}=K(x, t, u) \quad K \in \mathscr{L} .
$$

Definition 2. If a function $G=G(x, t, u) \in \mathscr{L}$ satisfies the linearised Equation of (3)

$$
\frac{\mathrm{d} G}{\mathrm{~d} t}=K^{\prime}(u)[G]
$$

we call it a symmetry of Equation (3), where $d / d t$ is the total $t$-derivative, $u$ is a solution of Equation (3) and $K^{\prime}(u)[G]$ is a Gateaux derivative of $K(u)$ in the direction $G$ with respect to $u$. 
Simple calculations show that the equation

$$
\frac{\partial G}{\partial t}=\llbracket K, G \rrbracket
$$

is equivalent to the linearised Equation (4), where $\llbracket$, $\rrbracket$ is defined as in Equation (2).

Denote the linear operators mapping $\mathscr{L}$ into itself by $L(\mathscr{L})$. Furthermore, we denote all differentiable operators that map $\mathbb{R}^{n} \times \mathbb{R} \times \mathscr{L}$ into $L(\mathscr{L})$ by $\mathscr{U}$. At the same time, we suppose $\Phi K=\Phi(x, t, u) K$ for $\Phi \in \mathscr{U}, K \in \mathscr{L},(x, t) \in \mathbb{R}^{N} \times \mathbb{R}, u \in \mathscr{L}$.

Definition 3. The Lie derivative $L_{K} \Phi \in \mathscr{U}$ of $\Phi$ with respect to $K$ is

$$
\left(L_{K} \Phi\right)=\Phi^{\prime}[K]-K^{\prime} \Phi+\Phi K^{\prime},
$$

where $\Phi \in \mathscr{U}, K \in \mathscr{L}$ and $\Phi^{\prime}[K]$ is the Gateaux derivative of the operator $\Phi(u)$ in the direction $K$ with respect to $u$.

Definition 4. If an operator $\Phi \in \mathscr{U}$ satisfies the following relation

$$
\Phi^{\prime}[\Phi K] S-\Phi^{\prime}[\Phi S] K=\Phi\left(\Phi^{\prime}[K] S-\Phi^{\prime}[S] K\right) \quad K, S \in \mathscr{L},
$$

then $\Phi$ is called a hereditary symmetry.

Definition 5. If an operator $\Phi \in \mathscr{U}$ maps one symmetry of Equation (3) into another symmetry of Equation (3), then $\Phi$ is a strong symmetry of Equation (3).

It is easy to verify that the relation

$$
\frac{\partial \Phi}{\partial t}+L_{K} \Phi=0
$$

is equivalent to $\Phi \in \mathscr{U}$ is a strong symmetry of Equation (3).

\section{Isospectral and Nonisospcetral Hierarchies of the gDNLS Equations}

In this section, from a matrix spectral problem with arbitrary parameter, we constitute the isospectral and nonisospectral gDNLS hierarchies.

Suppose that $T$ is the transpose of a matrix and

$$
\varsigma=\left(\begin{array}{cc}
-1 & 0 \\
0 & 1
\end{array}\right), \quad \delta=\left(\begin{array}{ll}
0 & 1 \\
1 & 0
\end{array}\right), e=\left(\begin{array}{ll}
1 & 0 \\
0 & 1
\end{array}\right)
$$

are the three Pauli matrices. Let us consider the following Lax pair $[30,31]$

$$
\phi_{x}=M \phi, \quad M=\left(\begin{array}{cc}
-\frac{1}{2}\left(\eta^{2}-\beta q r\right) & \eta q \\
\eta r & \frac{1}{2}\left(\eta^{2}-\beta q r\right)
\end{array}\right), \quad \phi=\left(\begin{array}{l}
\phi_{1} \\
\phi_{2}
\end{array}\right),
$$

and its time evolution

$$
\phi_{t}=N \phi, \quad N=\left(\begin{array}{cc}
A & B \\
C & -A
\end{array}\right),
$$

where $\eta$ is a spectral parameter and $q=q(x, t), r=r(x, t)$ are potential functions. We assume that $q(x, t)$ and $r(x, t)$ are smooth functions of variables $t$ and $x$; and their derivatives of any order with respect to $x$ vanish rapidly as $x \rightarrow \infty$. The compatibility condition, the zero curvature equation, reads 


$$
M_{t}-N_{x}+[M, N]=0,
$$

which yields

$$
\begin{gathered}
\frac{1}{2} \beta(q r)_{t}=A_{x}+\eta(r B-q C)+\eta \eta_{t}, \\
\eta q_{t}=B_{x}+2 \eta q A+\left(\eta^{2}-\beta q r\right) B-\eta_{t} q, \\
\eta r_{t}=C_{x}-2 \eta r A-\left(\eta^{2}-\beta q r\right) C-\eta_{t} r .
\end{gathered}
$$

Multiplying Equations (6b) and (6c) by $\beta r$ and $\beta q$, respectively, and adding the results together, we get

$$
\eta \beta(q r)_{t}=\beta r B_{x}+\beta q C_{x}+\beta\left(\eta^{2}-\beta q r\right)(r B-q C)-2 \beta q r \eta_{t}
$$

Substituting Equation (7) into Equation (6a) multiplied by $2 \eta$, and then integrating both sides, we have

$$
2 \eta A=2 \eta A_{0}-\beta \partial^{-1}(r, q)(\varsigma \partial+\beta q r e)\left(\begin{array}{c}
B \\
-C
\end{array}\right)-(2-\beta) \partial^{-1}(r, q)\left(\begin{array}{c}
B \\
-C
\end{array}\right) \eta^{2}-2\left(\beta \partial^{-1} q r+\eta^{2} x\right) \eta_{t},
$$

where $A_{0}$ is a integration constant. Finally, we obtain

$$
\begin{aligned}
\eta\left(\begin{array}{l}
q \\
r
\end{array}\right)_{t}= & L_{1} L_{2}\left(\begin{array}{c}
B \\
-C
\end{array}\right)+\eta^{2} L_{3}\left(\begin{array}{c}
B \\
-C
\end{array}\right)+2 \eta A_{0}\left(\begin{array}{c}
q \\
-r
\end{array}\right)-\eta_{t} L_{1}\left(\begin{array}{l}
q \\
r
\end{array}\right) \\
& -2 \eta^{2} \eta_{t}\left(\begin{array}{c}
x q \\
-x r
\end{array}\right),
\end{aligned}
$$

from Equations (6b) and (6c) by using Equation (8), where

$$
\begin{aligned}
& L_{1}=e+\beta\left(\begin{array}{c}
q \\
-r
\end{array}\right) \partial^{-1}(r, q), \quad L_{2}=-(\varsigma \partial+\beta q r e), \\
& L_{3}=e-(2-\beta)\left(\begin{array}{c}
q \\
-r
\end{array}\right) \partial^{-1}(r, q) .
\end{aligned}
$$

Setting $\eta_{t}=0, A_{0}=\frac{1}{2}(-1)^{n} \eta^{2 n}$,

$$
\left(\begin{array}{l}
B \\
C
\end{array}\right)=\sum_{j=1}^{n}(-1)^{n-j}\left(\begin{array}{l}
b_{j} \\
c_{j}
\end{array}\right) \eta^{2(n-j)+1},
$$

and comparing the coefficients of the same power of $\eta$ in Equation (9), we obtain

$$
\begin{aligned}
\left(\begin{array}{c}
q \\
r
\end{array}\right)_{t}=L_{1} L_{2}\left(\begin{array}{c}
b_{n} \\
-c_{n}
\end{array}\right), \\
\left(\begin{array}{c}
b_{1} \\
-c_{1}
\end{array}\right)=L_{3}^{-1}\left(\begin{array}{c}
q \\
-r
\end{array}\right), \\
\left(\begin{array}{c}
b_{j+1} \\
-c_{j+1}
\end{array}\right)=L_{3}^{-1} L_{1} L_{2}\left(\begin{array}{c}
b_{j} \\
-c_{j}
\end{array}\right), \quad(j=1,2, \cdots, n-1) .
\end{aligned}
$$


Through simple iteration, we arrive at the isospectral flow

$$
u_{t}=K_{n}=\Phi^{n}\left(\begin{array}{l}
q \\
-r
\end{array}\right)
$$

where $n$ is a positive integer and

$$
\Phi=\left(\begin{array}{ll}
\Phi_{11} & \Phi_{12} \\
\Phi_{21} & \Phi_{22}
\end{array}\right)
$$

with

$$
\begin{aligned}
& \Phi_{11}=\partial+(2-\beta) q r+(2-\beta) q_{x} \partial^{-1} r-\beta q \partial^{-1} r_{x}+2 \beta(1-\beta) q \partial^{-1} r^{2} q \\
& \Phi_{12}=(2-\beta) q_{x} \partial^{-1} q+\beta q \partial^{-1} q_{x}+2(1-\beta) q^{2}+2 \beta(1-\beta) q \partial^{-1} q^{2} r \\
& \Phi_{21}=(2-\beta) r_{x} \partial^{-1} r+\beta r \partial^{-1} r_{x}+2(1-\beta) r^{2}-2 \beta(1-\beta) r \partial^{-1} r^{2} q \\
& \Phi_{22}=-\partial+(2-\beta) q r+(2-\beta) r_{x} \partial^{-1} q-\beta r \partial^{-1} q_{x}-2 \beta(1-\beta) r \partial^{-1} q^{2} r
\end{aligned}
$$

Taking $u^{T}=(q, r)$, we can express $\Phi$ in vector forms

$$
\begin{aligned}
\Phi= & -\varsigma \partial+\frac{1}{2} \beta u^{T} \delta u e+(2-\beta) u_{x} \partial^{-1} u^{T} \delta+\beta \varsigma u \partial^{-1} u_{x}^{T} \varsigma \delta+2(1-\beta) u u^{T} \delta \\
& +\beta(\beta-1) \varsigma u \partial^{-1} u^{T} \delta u^{T} \delta u .
\end{aligned}
$$

Analogously, we can obtain the nonisospectral flow

$$
u_{t}=\sigma_{n}=\Phi^{n-1}\left(\begin{array}{c}
x q_{x}+\frac{1}{2} q \\
x r_{x}+\frac{1}{2} r
\end{array}\right)
$$

by taking $\eta_{t}=\frac{1}{2}(-1)^{n-1} \eta^{2 n-1}, A_{0}=0$.

The system in Equation (11) is integrable. It has infinite conservation laws and Hamilton structure. We prove that it is integrable in Liouville sense and gives the relation between infinite conservation laws and the Hamilton structure somewhere else.

The systems in Equations (11) and (13) are generalized systems. Many celebrated integrable systems can be derived from them by selecting different values of the arbitrary parameter $\beta$.

For example, when $n=2$, (11) becomes

$$
\begin{aligned}
& q_{t}=q_{x x}+2(2-\beta) q r q_{x}+2(1-\beta) q^{2} r_{x}+\beta(1-\beta) q^{3} r^{2}, \\
& r_{t}=-r_{x x}+2(2-\beta) q r r_{x}+2(1-\beta) r^{2} q_{x}-\beta(1-\beta) r^{3} q^{2} .
\end{aligned}
$$

We can deduce the three celebrated isospectral DNLS equations from it (Equation (14) has $n$-soliton and Wronski solutions with an arbitrary parameter, which will be shown in another paper).

- The isospectral Kaup-Newell (KN) equation

$$
\begin{aligned}
& q_{t}=q_{x x}+2\left(q^{2} r\right)_{x} \\
& r_{t}=-r_{x x}+2\left(q r^{2}\right)_{x}
\end{aligned}
$$

with $\beta=0$.

- The isospectal Chen-Lee-Liu (CLL) equation

$$
\begin{aligned}
& q_{t}=q_{x x}+2 q r q_{x}, \\
& r_{t}=-r_{x x}+2 q r r_{x}
\end{aligned}
$$

with $\beta=1$. 
- The isospectral Gerdjikov-Ivanov (GI) equation

$$
\begin{aligned}
& q_{t}=q_{x x}-2 q^{2} r_{x}-2 q^{3} r^{2}, \\
& r_{t}=-r_{x x}-2 r^{2} q_{x}+2 q^{2} r^{3},
\end{aligned}
$$

with $\beta=2$.

When $n=2$, Equation (13) becomes

$$
\begin{aligned}
q_{t}= & x q_{x x}+2(2-\beta) x q r q_{x}+\frac{3}{2} q_{x}+\beta q \partial^{-1} q_{x} r \\
& +2(1-\beta)\left(x r_{x}+r\right) q^{2}+\beta(1-\beta)\left(q^{3} r^{2} x+q \partial^{-1} q^{2} r^{2}\right), \\
r_{t}= & -x r_{x x}+2(2-\beta) x q r r_{x}-\frac{3}{2} r_{x}+\beta r \partial^{-1} r_{x} q \\
& +2(1-\beta)\left(x q_{x}+q\right) r^{2}-\beta(1-\beta)\left(r^{3} q^{2} x+r \partial^{-1} q^{2} r^{2}\right) .
\end{aligned}
$$

We can also deduce the three celebrated nonisospectral DNLS equations. For example,

- The nonisospectral KN equation

$$
\begin{aligned}
& q_{t}=x q_{x x}+2 x\left(q^{2} r\right)_{x}+\frac{3}{2} q_{x}+2 q^{2} r, \\
& r_{t}=-x r_{x x}+2 x\left(q r^{2}\right)_{x}-\frac{3}{2} r_{x}+2 q r^{2}
\end{aligned}
$$

with $\beta=0$.

- The nonisospectral CLL equation

$$
\begin{aligned}
& q_{t}=x q_{x x}+2 x q r q_{x}+\frac{3}{2} q_{x}+q \partial^{-1} q_{x} r, \\
& r_{t}=-x r_{x x}+2 x q r r_{x}-\frac{3}{2} r_{x}+r \partial^{-1} q r_{x},
\end{aligned}
$$

with $\beta=1$.

- The nonisospectral GI equation

$$
\begin{aligned}
& q_{t}=x q_{x x}-2 x q^{2} r_{x}-2 x q^{3} r^{2}+\frac{3}{2} q_{x}+2 q \partial^{-1} q_{x} r-2 q^{2} r-2 q \partial^{-1} q^{2} r^{2}, \\
& r_{t}=-x r_{x x}-2 x r^{2} q_{x}+2 x q^{2} r^{3}-\frac{3}{2} r_{x}+2 r \partial^{-1} q r_{x}-2 q r^{2}+2 r \partial^{-1} q^{2} r^{2},
\end{aligned}
$$

with $\beta=2$.

When $n=3$, Equation (11) becomes

$$
\begin{aligned}
q_{t}= & q_{x x x}+3(2-\beta)\left(q_{x} q\right)_{x} r+6(1-\beta) q q_{x} r_{x}+3\left(6-6 \beta+\beta^{2}\right) q^{2} q_{x} r^{2} \\
& +6(1-\beta)(2-\beta) q^{3} r r_{x}+2 \beta(1-\beta)(2-\beta) q^{4} r^{3} \\
r_{t}= & r_{x x x}-3(2-\beta) q\left(r r_{x}\right)_{x}-6(1-\beta) q_{x} r r_{x}+3\left(6-6 \beta+\beta^{2}\right) q^{2} r^{2} r_{x} \\
& +6(1-\beta)(2-\beta) q q_{x} r^{3}-2 \beta(1-\beta)(2-\beta) q^{3} r^{4} .
\end{aligned}
$$

Through taking the different values of $\beta$ and $r$, we can get the following different types of equations.

- The modified Korteweg-de Vries (mKdV) equation

$$
q_{t}=q_{x x x}-6 q^{2} q_{x}
$$

with $\beta=2$ and $r=1$. 
- The Sharma-Tasso-Olever (STO) equation

$$
q_{t}=q_{x x x}+3\left(q q_{x}\right)_{x}+3 q^{2} q_{x}
$$

with $\beta=1$ and $r=1$.

- A new equation

$$
q_{t}=q_{x x x}-6 i|q|^{2} q_{x x}-6 i q^{*} q_{x}^{2}-6 i q\left|q_{x}\right|^{2}-18|q|^{4} q_{x}-12 q^{2}|q|^{2} q_{x}
$$

with $\beta=0$ and $r=-i q$.

As far as we known, Equation (20) is a new integrable equation with fifth-order nonlinear term. Since it is a special case of the system in Equation (11), it has infinite conservation laws and Hamilton Structure. It also has two symmetries and these symmetries have an infinite dimensional Lie algebra structure.

If we replace $\eta$ with $i \lambda$, as the process given in [32], we can restrict Equations (14) and (19) to nonlocal soliton systems. Recent research shows that these nonlocal soliton systems are not only integrable [33] but also can be solved by inverse scattering transformation [32,34].

\section{A $\tau$-Symmetry Algebra of the gDNLS Soliton Hierarchy}

In this section, we prove that the obtained isospectral flow in Equation (11) and nonisospectral flow in Equation (13) are symmetries of Equation (11) and they have an infinite dimensional algebra structure. For the two types of symmetries related to the recursion operator $\Phi$, firstly we prove the integro-differential operator $\Phi$ is not only a hereditary symmetry operator but also a strong symmetry operator.

Lemma 1. For arbitrary $f, g \in C^{\infty}$, let

$$
\begin{aligned}
w_{1}= & 2(1-\beta) \varsigma\left(g u^{T} \delta f_{x}-f u^{T} \delta g_{x}\right)-\beta\left(g_{x}^{T} \varsigma \delta u f-f_{x}^{T} \varsigma \delta u g\right)+\beta \varsigma u\left(f_{x}^{T} \delta g-g_{x}^{T} \delta f\right) \\
& -(2-\beta) \varsigma\left(f_{x} u^{T} \delta g-g_{x} u^{T} \delta f\right)+2(1-\beta) u\left(f_{x}^{T} \varsigma \delta g-g_{x}^{T} \varsigma \delta f\right) .
\end{aligned}
$$

Then, we have $w_{1}=0$.

Proof. Simple calculations show that, for arbitrary $f, g, u \in C^{\infty}$, the following identity relations

$$
\begin{aligned}
& u^{T} \varsigma \delta f_{x}=-f_{x}^{T} \varsigma \delta u, \quad u^{T} \delta f_{x}=f_{x}^{T} \delta u, \quad f u_{x}^{T} \varsigma \delta g-g u_{x}^{T} \varsigma \delta f=\varsigma f u_{x}^{T} \delta g-\varsigma g u_{x}^{T} \delta f, \\
& \varsigma f_{x} u^{T} \delta g-\varsigma g u^{T} \delta f_{x}=u f_{x}^{T} \varsigma \delta g, \quad \varsigma g u^{T} \delta f_{x}-\varsigma u f_{x}^{T} \delta g=f_{x}^{T} \varsigma \delta u g-u f_{x}^{T} \varsigma \delta g,
\end{aligned}
$$

hold. Using the above relations, we conclude that $w_{1}=0$.

Lemma 2. For arbitrary $f, g \in C^{\infty}$, let

$$
w_{3}=(2-\beta)^{2} w_{35}+\beta(2-\beta)\left(w_{36}+\frac{1}{2} w_{33}\right)+2 \beta(1-\beta)\left(w_{34}+3 w_{32}\right)-2 \beta^{2} w_{31},
$$

where

$$
\begin{aligned}
& w_{31}=\varsigma u \partial^{-1} f^{T} \varsigma \delta g u^{T} \delta u_{x}, \quad w_{32}=\varsigma u \partial^{-1} u_{x}^{T} \varsigma \delta f u^{T} \delta g-\varsigma u \partial^{-1} u_{x}^{T} \varsigma \delta g u^{T} \delta f, \\
& w_{33}=u^{T} \delta u u^{T} \delta g f-u^{T} \delta u u^{T} \delta f g-\varsigma u \partial^{-1} f_{x}^{T} \varsigma \delta g u^{T} \delta u+\varsigma u \partial^{-1} g_{x}^{T} \varsigma \delta f u^{T} \delta u,
\end{aligned}
$$




$$
\begin{aligned}
w_{34}= & \varsigma u \partial^{-1} u^{T} \delta f g_{x}^{T} \varsigma \delta u-\varsigma u \partial^{-1} u^{T} \delta g f_{x}^{T} \varsigma \delta u-\varsigma u \partial^{-1} u^{T} \varsigma \delta g u_{x}^{T} \delta f+\varsigma u \partial^{-1} u^{T} \varsigma \delta f u_{x}^{T} \delta g \\
& +f u^{T} \delta g u^{T} \delta u-g u^{T} \delta f u^{T} \delta u-\varsigma u \partial^{-1} u^{T} \varsigma \delta g u^{T} \delta f_{x}+\varsigma u \partial^{-1} u^{T} \varsigma \delta f u^{T} \delta g_{x}, \\
w_{35}= & u_{x} \partial^{-1} u^{T} \delta f_{x} \partial^{-1} u^{T} \delta g-u_{x} \partial^{-1} u^{T} \delta g_{x} \partial^{-1} u^{T} \delta f-u_{x} u^{T} \delta f \partial^{-1} u^{T} \delta g \\
& +u_{x} u^{T} \delta g \partial^{-1} u^{T} \delta f-u_{x} \partial^{-1} u_{x}^{T} \delta g \partial^{-1} u^{T} \delta f+u_{x} \partial^{-1} u_{x}^{T} \delta f \partial^{-1} u^{T} \delta g \\
w_{36}= & \varsigma u \partial^{-1} u_{x}^{T} \varsigma \delta f_{x} \partial^{-1} u^{T} \delta g-\varsigma u \partial^{-1} u_{x}^{T} \varsigma \delta g_{x} \partial^{-1} u^{T} \delta f-\varsigma u u_{x}^{T} \varsigma \delta f \partial^{-1} u^{T} \delta g \\
& +\varsigma u u_{x}^{T} \varsigma \delta g \partial^{-1} u^{T} \delta f-\varsigma u \partial^{-1} u_{x x}^{T} \varsigma \delta g \partial^{-1} u^{T} \delta f+\varsigma u \partial^{-1} u_{x x}^{T} \varsigma \delta f \partial^{-1} u^{T} \delta g .
\end{aligned}
$$

Then, we have $w_{3}=0$.

Proof. It is easy to verify that

$$
w_{35}=0, \quad \frac{1}{2} w_{33}=w_{34}=w_{36}=w_{31}=-w_{32} .
$$

Through simple calculation, we conclude that $w_{3}=0$.

Lemma 3. For arbitrary $f, g \in C^{\infty}$, let

$$
w_{5}=\beta(1-\beta)(2-\beta)\left(w_{51}+w_{52}+w_{53}+w_{54}\right),
$$

where

$$
\begin{aligned}
& w_{51}=\varsigma u\left(\partial^{-1} u_{x}^{T} \delta f u^{T} \delta u \partial^{-1} u^{T} \delta g-\partial^{-1} u_{x}^{T} \delta g u^{T} \delta u \partial^{-1} u^{T} \delta f\right) \\
& w_{52}=2 \varsigma u\left(\partial^{-1} u^{T} \delta f u_{x}^{T} \delta u \partial^{-1} u^{T} \delta g-\partial^{-1} u^{T} \delta g u_{x}^{T} \delta u \partial^{-1} u^{T} \delta f\right) \\
& w_{53}=\varsigma u\left(\partial^{-1} u^{T} \delta g_{x} u^{T} \delta u \partial^{-1} u^{T} \delta f-\partial^{-1} u^{T} \delta f_{x} u^{T} \delta u \partial^{-1} u^{T} \delta g\right), \\
& w_{54}=\varsigma u\left(u^{T} \delta g u^{T} \delta g u^{T} \delta u \partial^{-1} u^{T} \delta f-u^{T} \delta g u^{T} \delta f u^{T} \delta u \partial^{-1} u^{T} \delta g\right) .
\end{aligned}
$$

Then, we have $w_{5}=0$.

Proof. It is easy to verify that

$$
w_{51}+w_{52}+w_{53}+w_{54}=0
$$

and so $w_{5}=0$.

Theorem 1. The integro-differential operator $\Phi$ is hereditary.

Proof. Noticing that $u^{T} \varsigma \delta u=0$, we have

$$
\Phi\left(\Phi^{\prime}[f] g-\Phi^{\prime}[g] f\right)-\left(\Phi^{\prime}[\Phi f] g-\Phi^{\prime}[\Phi g] f\right)=w_{1}+w_{3}+w_{5}
$$

with arbitrary $f, g \in C^{\infty}$. By Lemmas 1, 2 and 3, we know that

$$
\Phi\left(\Phi^{\prime}[f] g-\Phi^{\prime}[g] f\right)=\left(\Phi^{\prime}[\Phi f] g-\Phi^{\prime}[\Phi g] f\right) .
$$

The hereditary operator $\Phi$ ensures that the properties of strong operators can be inherited from one equation to the next equation in Equation (11), i.e., if $\Phi$ is a strong symmetry operator of the first equation in the system in Equation (11), it is also the strong symmetry operator of the every equation in the system in Equation (11). 
Lemma 4. The integro-differential operator $\Phi$ satisfies

$$
\Phi^{\prime}\left[K_{m}\right]=\left[K_{m}^{\prime}, \Phi\right]
$$

and it is a strong symmetry of the gDNLS soliton hierarchy in Equation (11).

Proof. It is easy to verify

$$
\begin{aligned}
\Phi^{\prime}[-\varsigma u]= & (-\varsigma u)^{\prime} \Phi-\Phi(-\varsigma u)^{\prime} \\
= & \beta(1-\beta) \varsigma u \partial^{-1} u^{T} \varsigma \delta u^{T} \delta u-(2-\beta) \varsigma u_{x} \partial^{-1} u^{T} \delta-2(1-\beta) u u^{T} \varsigma \delta \\
& -(2-\beta) u_{x} \partial^{-1} u^{T} \varsigma \delta-\beta u \partial^{-1} u_{x}^{T} \varsigma \delta-\beta \varsigma u \partial^{-1} u_{x}^{T} \delta-2(1-\beta) \varsigma u u^{T} \delta \\
& -\beta(\beta-1) u \partial^{-1} u^{T} \delta u^{T} \delta u .
\end{aligned}
$$

The above equality and Theorem 1 show that $\Phi$ is a hereditary and strong symmetry operator for the equation $u_{t}=K_{0}$. Thus, $\Phi$ is a strong symmetry operator for $u_{t}=\Phi^{m} K_{0}=K_{m}$ and the proof has been complete.

\section{Lemma 5.}

$$
\Phi^{\prime}\left[\sigma_{n}\right]+\Phi \sigma_{n}^{\prime}-\sigma_{n}^{\prime} \Phi=\Phi^{n}, \quad n=1,2, \cdots
$$

Proof. When $n=1$, we have

$$
\Phi^{\prime}\left[\sigma_{1}\right]+\Phi \sigma_{1}^{\prime}-\sigma_{1}^{\prime} \Phi=\Phi
$$

through simple calculation. Assume that

$$
\Phi^{\prime}\left[\sigma_{m}\right]+\Phi \sigma_{m}^{\prime}-\sigma_{m}^{\prime} \Phi=\Phi^{m}
$$

we have

$$
\begin{aligned}
& \left(\Phi^{m+1}-\Phi \sigma_{m+1}^{\prime}+\sigma_{m+1}^{\prime} \Phi\right) f \\
= & \Phi^{m+1} f-\Phi\left(\Phi \sigma_{m}\right)^{\prime} f+\left(\Phi \sigma_{m}\right)^{\prime} \Phi f \\
= & \Phi^{m+1} f-\Phi\left(\Phi^{\prime}[f] \sigma_{m}+\Phi \sigma_{m}^{\prime}[f]\right)+\Phi^{\prime}[\Phi f] \sigma_{m}+\Phi \sigma_{m}^{\prime}[\Phi f] \\
= & \Phi^{m+1} f-\Phi \Phi^{\prime}[f] \sigma_{m}-\Phi\left(\sigma_{m}^{\prime} \Phi+\Phi^{m}-\Phi^{\prime}\left[\sigma_{m}\right]\right) f+\Phi^{\prime}[\Phi f] \sigma_{m}+\Phi \sigma_{m}^{\prime}[\Phi f] \\
= & \Phi \Phi^{\prime}\left[\sigma_{m}\right] f+\Phi^{\prime}[\Phi f] \sigma_{m}-\Phi \Phi^{\prime}[f] \sigma_{m} \\
= & \Phi^{\prime}\left[\Phi \sigma_{m}\right] f \\
= & \Phi^{\prime}\left[\sigma_{m+1}\right] f
\end{aligned}
$$

where the fact that $\Phi$ is a hereditary symmetry operator has been used. Thus, Equation (22) holds for any positive integer $n$.

Lemma 5 gives a relation between the recursion operator $\Phi$ and $\tau$-symmetries. It is very important in the following computation.

Theorem 2. The two flows $K_{m}$ in Equation (11) and $\sigma_{m}$ in Equation (13) deduced from the matrix spectral problem of the gDNLS soliton equation satisfy the following commutator relations:

$$
\begin{gathered}
\llbracket K_{m}, K_{n} \rrbracket=0, \\
\llbracket K_{m}, \sigma_{n} \rrbracket=m K_{m+n-1}, \\
\llbracket \sigma_{m}, \sigma_{n} \rrbracket=(m-n) \sigma_{m+n-1}, \quad m, n=1,2, \cdots .
\end{gathered}
$$

In other words, these two flows form an infinite-dimensional Lie algebra. 
Proof. We only prove Equation (23b), as the other two identities can be proven similarly.

Firstly, we prove the following identity

$$
\llbracket K_{m}, \sigma_{1} \rrbracket=m K_{m} .
$$

By simple calculation, we have

$$
\begin{aligned}
\llbracket K_{0}, \sigma_{1} \rrbracket & =K_{0}^{\prime}\left[\sigma_{1}\right]-\sigma_{1}^{\prime}\left[K_{0}\right] \\
& =\left(\begin{array}{ll}
1 & 0 \\
0 & -1
\end{array}\right)\left(\begin{array}{l}
x q_{x}+\frac{q}{2} \\
x r_{x}+\frac{r}{2}
\end{array}\right)-\left(\begin{array}{ll}
x \partial+\frac{1}{2} & 0 \\
0 & x \partial+\frac{1}{2}
\end{array}\right)\left(\begin{array}{l}
q \\
-r
\end{array}\right) \\
& =0 .
\end{aligned}
$$

Thus, Equation (24) is right in the case of $m=0$.

Suppose that Equation (24) is true in the case of $m-1$, i.e.,

$$
\llbracket K_{m-1}, \sigma_{1} \rrbracket=(m-1) K_{m-1},
$$

and then

$$
\begin{aligned}
\llbracket K_{m}, \sigma_{1} \rrbracket & =\llbracket \Phi K_{m-1}, \sigma_{1} \rrbracket \\
& =\Phi^{\prime}\left[\sigma_{1}\right] K_{m-1}+\Phi K_{m-1}^{\prime}\left[\sigma_{1}\right]-\sigma_{1}^{\prime}\left[K_{m}\right] \\
& =\left(\Phi+\sigma_{1}^{\prime} \Phi-\Phi \sigma_{1}^{\prime}\right) K_{m-1}+\Phi K_{m-1}^{\prime}\left[\sigma_{1}\right]-\sigma_{1}^{\prime}\left[K_{m}\right] \\
& =K_{m}+\Phi \llbracket K_{m-1}, \sigma_{1} \rrbracket \\
& =m K_{m} .
\end{aligned}
$$

Thus, for any positive integer $m$, Equation (24) holds.

Finally, let us think about the general identity in Equation (23b) by using the similar method. We already know that the identity in Equation (23b) is true in the case of $n=1$ from Equation (24). Suppose that Equation (23b) is true in the case of $n-1$, i.e.,

$$
\llbracket K_{m}, \sigma_{n-1} \rrbracket=m K_{m+n-2} .
$$

It is easy to find that

$$
\begin{aligned}
\llbracket K_{m}, \sigma_{n} \rrbracket & =\llbracket K_{m}, \Phi \sigma_{n-1} \rrbracket \\
& =K_{m}^{\prime}\left[\Phi \sigma_{n-1}\right]-\Phi^{\prime}\left[K_{m}\right] \sigma_{n-1}-\Phi \sigma_{n-1}^{\prime}\left[K_{m}\right] \\
& =K_{m}^{\prime}\left[\Phi \sigma_{n-1}\right]-\left(K_{m}^{\prime} \Phi-\Phi K_{m}^{\prime}\right) \sigma_{n-1}-\Phi \sigma_{n-1}^{\prime}\left[K_{m}\right] \\
& =\Phi \llbracket K_{m}, \sigma_{n-1} \rrbracket \\
& =m K_{m+n-1} .
\end{aligned}
$$

Theorem 2 tells us that the isospectral and nonisospectral flows obtained in Section 3 are $K$-symmetries and $\tau$-symmetries of Equation (11), respectively. They constitute an infinite dimensional Lie algebra.

Corollary 1. It is interesting that the vector field $\sigma_{2}(u)$ is actually a master symmetry, i.e., the other symmetries can be generated by it through the following relations:

$$
K_{s+1}=\frac{1}{s} \llbracket K_{s}, \sigma_{2} \rrbracket,
$$




$$
\sigma_{s+1}=\frac{1}{s-2} \llbracket \sigma_{s}, \sigma_{2} \rrbracket \quad s \neq 2
$$

Finally, let us consider combinations of the two kinds of symmetries $K_{m}, \sigma_{n}$ and the time variable $t$. We define new functions $\tau_{0}^{m}$ and $\tau_{n}^{m}$ as

$$
\begin{gathered}
\tau_{0}^{m}=m t K_{m}+\sigma_{1}, \\
\tau_{n}^{m}=\Phi^{n} \tau_{0}^{m}=m t K_{m+n}+\sigma_{n+1} .
\end{gathered}
$$

Theorem 3. The new functions $\tau_{n}^{m}$ are all symmetries of Equation (11), i.e.,

$$
\left(\tau_{n}^{m}\right)_{t}=K_{m}^{\prime}\left[\tau_{n}^{m}\right], \quad m=0,1,2, \cdots ; n=1,2, \cdots .
$$

Proof. Since we have already know that $\Phi$ is a strong symmetry operator of Equation (11), it just proves

$$
\left(\tau_{0}^{m}\right)_{t}=K_{m}^{\prime}\left[\tau_{0}^{m}\right]
$$

Through Equation (25a), is is easy to find

$$
\begin{aligned}
\left(\tau_{0}^{m}\right)_{t} & =m K_{m}+m t K^{\prime}\left[u_{t}\right]+\sigma_{1}^{\prime}\left[u_{t}\right] \\
& =m K_{m}+m t K^{\prime}\left[K_{m}\right]+\sigma_{1}^{\prime}\left[K_{m}\right] \\
& =m t K^{\prime}\left[K_{m}\right]+K_{m}^{\prime}\left[\sigma_{1}\right] \\
& =K_{m}^{\prime}\left[\tau_{0}^{m}\right] .
\end{aligned}
$$

Theorem 3 shows that $\tau_{n}^{m}$ is another kind symmetry of Equation (11). In fact, it is the special linear combination of $K$-symmetry and $\tau$-symmetry.

Theorem 4. $K_{m}$ and $\tau_{m}^{n}, \quad m \geq 0$ are two sets of symmetries of every equation in the isospectral gDNLS hierarchy. They satisfy the following relations

$$
\begin{gathered}
\llbracket K_{m}, K_{n} \rrbracket=0, \\
\llbracket K_{m}, \tau_{n}^{l} \rrbracket=m K_{m+n-1}, \\
\llbracket \tau_{l}^{m}, \tau_{n}^{m} \rrbracket=(l-n) \tau_{l+n-1}^{m} .
\end{gathered}
$$

Thus, these symmetries also form an infinite-dimensional Lie algebra.

Proof. It is easy to find that

$$
\begin{aligned}
\llbracket \tau_{l}^{m}, \tau_{n}^{m} \rrbracket & =\llbracket m t K_{m+l}+\sigma_{l+1}, m t K_{m+n}+\sigma_{n+1} \rrbracket \\
& =m t\left\{\llbracket K_{m+l}, \sigma_{n+1} \rrbracket+\llbracket \sigma_{l+1}, K_{m+n} \rrbracket\right\}+\llbracket \sigma_{l+1}, \sigma_{n+1} \rrbracket \\
& =m t\left\{(m+l) K_{m+n+l}-(m+n) K_{m+n+l}\right\}+(l-n) \sigma_{n+l+1} \\
& =(l-n)\left(m t K_{m+n+l}+\sigma_{n+l+1}\right) \\
& =(l-n) \tau_{n+l}^{m}
\end{aligned}
$$

by Equations (23) and (25b). Thus, Equation (27c) holds and the other two equalities can be proven similarly.

$\tau$-symmetries should exist for nonlocal integrable equations developed based on the IST theory [32,34]. Evolution equations generated from $\tau$-symmetry vector fields are normally integrable 
based on the IST, but there may not be infinitely many conservation laws for them. Only the first several (normally two) $\tau$-symmetries are local, and all others in the $\tau$-symmetry hierarchy are not local.

The conclusions of Theorems 1-4 and Lemma 4 are generalized because of the arbitrary parameter $\beta$. If we take the special values of the parameter $\beta$, the conclusions are also true.

For example, when $\beta=0$, the recursion operator $\Phi$ becomes

$$
\Phi_{0}=-\varsigma \partial+2 u_{x} \partial^{-1} u^{T} \delta+2 u u^{T} \delta .
$$

$\Phi_{0}$ is a hereditary and strong symmetry operator of the KN Equation (15) and it has two sets of symmetries

$$
K_{m}=\Phi_{0}^{m}(-\varsigma u), \quad \tau_{n}^{m}=m t \Phi_{0}^{m+n}(-\varsigma u)+\Phi_{0}^{n}\left(x u_{x}+\frac{1}{2} u\right) \quad m=0,1,2, \cdots, \quad n=1,2, \cdots .
$$

When $\beta=1$, the recursion operator $\Phi$ becomes

$$
\Phi_{1}=-\varsigma \partial+\frac{1}{2} u^{T} \delta u e+u_{x} \partial^{-1} u^{T} \delta+\varsigma u \partial^{-1} u_{x}^{T} \delta \delta .
$$

$\Phi_{1}$ is a hereditary and strong symmetry operator of the CLL Equation (16) and it has two sets of symmetries

$$
K_{m}=\Phi_{1}^{m}(-\varsigma u), \quad \tau_{n}^{m}=m t \Phi_{1}^{m+n}(-\varsigma u)+\Phi_{1}^{n}\left(x u_{x}+\frac{1}{2} u\right) \quad m=0,1,2, \cdots, \quad n=1,2, \cdots .
$$

When $\beta=2$, the recursion operator $\Phi$ becomes

$$
\Phi_{2}=-\varsigma \partial+u^{T} \delta u e+2 \varsigma u \partial^{-1} u_{x}^{T} \varsigma \delta-2 u u^{T} \delta+2 \varsigma u \partial^{-1} u^{T} \delta u^{T} \delta u .
$$

$\Phi_{2}$ is a hereditary and strong symmetry operator of the GI Equation (17) and it has two sets of symmetries

$$
K_{m}=\Phi_{2}^{m}(-\varsigma u), \quad \tau_{n}^{m}=m t \Phi_{2}^{m+n}(-\varsigma u)+\Phi_{2}^{n}\left(x u_{x}+\frac{1}{2} u\right) \quad m=0,1,2, \cdots, \quad n=1,2, \cdots .
$$

\section{Conclusions}

In general, the integrability of the gDNLS equation is researched in this paper. The isospectral and nonisospectral hierarchies are derived from its Lax pair. Through taking different values of the arbitrary parameter, the gDNLS equation could be reduced to the KN equation, the CLL equation, the GI equation, the $\mathrm{mKdV}$ equation, the STO equation and so on. Two kinds of symmetries, $K$-symmetries and $\tau$-symmetries, are deduced from the corresponding isospectral and nonisospectral hierarchies. The recursion operator $\Phi$ related to these symmetries with an arbitrary parameter is proven to be a hereditary and strong symmetry operator of the whole isospectral hierarchy. Finally, we find that those two kinds of symmetries form an infinite-dimensional Lie algebra.

Author Contributions: These authors contributed equally to this work.

Funding: The work was supported in part by NSFC under the grants 11771186, 11671177, 11371326, 11571079, 11371086 and 13713611; NSF under the grant DMS-1664561; the Jiangsu Qing Lan Project (2014); Six Talent Peaks Project of Jiangsu Province (2016-JY-08); and the Distinguished Professorships by Shanghai University of Electric Power, China and North-West University, South Africa.

Conflicts of Interest: The authors declare that there is no conflict of interests regarding the publication of this article. 


\section{References}

1. Ablowitz, M.J.; Kaup, D.J.; Newell, A.C.; Segur, H. The Inverse Scattering Transform-Fourier Analysis for Nonlinear Problems. Stud. Appl. Math. 1974, 53, 249-315. [CrossRef]

2. Zhang, J.B.; Zhang, D.J.; Chen, D.Y. Exact Solutions to a Mixed Toda Lattice Hierarchy Through the Inverse Scattering Transformation. J. Phys. A Math. Gen. 2011, 44, 115201. [CrossRef]

3. Magri, F. A Simple Model of the Integrable Hamiltonian Equation. J. Math. Phys. 1978, 19, 1156-1162. [CrossRef]

4. Ma, W.X.; Chen, M. Hamiltonian and Quasi-Hamiltonian Structrues Associated with Semi-direct Sums of Lie Algebras. J. Phys. A Math. Gen. 2006, 39, 10787-10801. [CrossRef]

5. Ma, W.X. A Discrete Variational Identity on Semi-direct Sums of Lie Algebras. J. Phys. A Math. Gen. 2007, 40, 15055-15070. [CrossRef]

6. Ma, W.X. Conservation Laws of Discrete Evolution Equations by Symmetries and Adjoint Symmetries. Symmetry 2015, 7, 714-725. [CrossRef]

7. Ma, W.X. Conservation Laws by Symmetries and Adjoint Symmetries. Discret. Contin. Dyn. Syst. Ser. S 2018, 11, 707-721. [CrossRef]

8. Wadati, M. Invariances and Conservation Laws of the Korteweg-de Vries Equation. Stud. Appl. Math. 1978 , 59, 153-186. [CrossRef]

9. Alberty, J.M.; Koikawa, T.; Sasaki, R. Canonical Structure of Soliton Equations. I. Physica D 1982, 5, 43-65. [CrossRef]

10. Yang, J.Y.; Ma, W.X. Conservation Laws of a Perturbed Kaup-Newell Equation. Mod. Phys. Lett. B 2016, 30, 1650381. [CrossRef]

11. Zhang, J.B.; Ji, J.; Chen, D.Y. Conservation Laws and Symmetries of the Levi Equation. Commun. Theor. Phys. 2008, 50, 1261-1264.

12. Zhang, J.B.; Ji, J.; Yao, Y.Q. From the Conservation Laws to the Hamiltonian Structures of Discrete Soliton Systems. Phys. Scr. 2011, 84, 015001. [CrossRef]

13. Zhang, J.B.; Gongye, Y.Y.; Ma, W.X. Conservation Laws and $\tau$-Symmetry Algebra of the Gerdjikov-Ivanov Soliton Hierarchy. Bull. Malays. Math. Sci. Soc. 2018. [CrossRef]

14. Li, Y.S.; Zhu, G.C. New Set of Symmetries of the Integrable Equations, Lie Algebra and Non-isospectral Evolution Equations. II. AKNS System. J. Phys. A Math. Gen. 1986, 19, 3713-3725.

15. Li, Y.S.; Zhu, G.C. New Set of Symmetries of the Integrable Equations, Lie Algebra and Non-isospectral Evolution Equations (I). Sci. China Ser. A 1987, 30, 1243-1250.

16. Cheng, Y.; Li, Y.S. Symmetries and Constants of Motion for New AKNS Hierarchies. J. Phys. A Math. Gen. 1987, 20, 1951-1959. [CrossRef]

17. Li, Y.S.; Cheng, Y. Symmetries and Constants of Motion for New KdV Hierarchies. Sci. China Ser. A 1988, 31, 769-226.

18. Tu, G.Z. The Lie Algebraic Structure of Symmetries Generated by Hereditary Symmetries. J. Phys. A Math. Gen. 1988, 21, 1951-1957.

19. Ma, W.X. K-symmetries and $\tau$-symmetries of Evolution Equations and Their Lie Algebras. J. Phys. A Math. Gen. 1990, 23, 2707-2716. [CrossRef]

20. Ma, W.X. Generators of Vector Fields and Time Dependent Symmetries of Evolution Equations. Sci. China Ser. A 1991, 34, 769-782.

21. Ma, W.X.; Fuchssteiner, B. Algebraic Structure of Discrete Zero Curvature Equations and Master Symmetries of Discrete Evolution Equations. J. Math. Phys. 1999, 40, 2400-2418. [CrossRef]

22. Zhang, D.J.; Chen, S.T. Symmetries for the Ablowitz-Ladik Hierarchy: Part I. Four-Potential Case. Stud. Appl. Math. 2010, 125, 393-418. [CrossRef]

23. Zhang, D.J.; Chen, S.T. Symmetries for the Ablowitz-Ladik Hierarchy: Part II. Integrable Discrete Nonlinear Schrödinger Equations and Discrete AKNS Hierarchy. Stud. Appl. Math. 2010, 125, 419-443. [CrossRef]

24. Fu, W.; Huang, L.; Tamizhmani, K.M.; Zhang, D.J. Integrability Properties of the Differential-difference Kadomtsev-Petviashvili Hierarchy and Continuum limits. Nonlinearity 2013, 26, 3197-3229. [CrossRef]

25. Kaup, D.J.; Newell, A.C. An Exact Solution for a Derivative Nonlinear Schrödinger Equation. J. Math. Phys. 1978, 19, 798-801. [CrossRef] 
26. Chen, H.H.; Lee, Y.C.; Liu, C.S. Integrability of Nonlinear Hamiltonian Systems by Inverse Scattering Method. Phys. Scr. 1979, 20, 490-492. [CrossRef]

27. Kakei, S.; Sasa, N.; Satsuma, J. Bilinearization of a Generalized Derivative Nonlinear Schrödinger Equation. J. Phys. Soc. Jpn. 1995, 64, 1519-1523. [CrossRef]

28. Gerdjikov, V.S.; Ivanov, M.I. The Quadratic Bundle of General Form and the Nonlinear Evolution Equations. II. Hierarchies of Hamiltonian Structures. Bul. J. Phys. 1983, 10, 130-143.

29. Zhang, J.B.; Gongye, Y.Y.; Chen, S.T. Soliton Solutions to the Coupled Gerdjikov-Ivanov Equation with Rogue-wave-like Phenomena. Chin. Phys. Lett. 2017, 34, 090201. [CrossRef]

30. Geng, X.G.; Ma, W.X. A Generalized Kaup-Newell Spectral Problem, Soliton Equations and Finite-dimensional Integrable Systems. Il Nuovo Cimento A 1995, 108, 477-486. [CrossRef]

31. Fan, E.G. Integrable Systems of Derivative Nonlinear Schrödinger Type and Their Multi-Hamiltonian Structure. J. Phys. A Math. Gen. 2001, 34, 513-519. [CrossRef]

32. Ablowitz, M.J.; Musslimani, Z.H. Inverse Scattering Transform for the Integrable Nonlocal Nonlinear Schrödinger Equation. Nonlinearity 2016, 29, 915-946. [CrossRef]

33. Ablowitz, M.J.; Musslimani, Z.H. Integrable Nonlocal Nonlinear Equation. Stud. Appl. Math. 2016, 139, 7-59. [CrossRef]

34. Ablowitz, M.J.; Musslimani, Z.H. Integrable Nonlocal Nonlinear Schrödinger Equation. Phys. Rev. Lett. 2013, 110, 064105. [CrossRef] [PubMed]

(C) 2018 by the authors. Licensee MDPI, Basel, Switzerland. This article is an open access article distributed under the terms and conditions of the Creative Commons Attribution (CC BY) license (http://creativecommons.org/licenses/by/4.0/). 